\title{
Utilization of Internal Bracing in Elbow Medial UCL Stabilization: From Biomechanics to Clinical Application and Patient Outcomes
}

\author{
Andre Anvari \\ Amir Fathi \\ loanna K Bolia (ID) \\ Eric Piatt \\ Laith $\mathrm{K}$ Hasan \\ Aryan Haratian \\ Alexander E Weber \\ Frank A Petrigliano \\ USC Epstein Family Center for Sports \\ Medicine at Keck Medicine of USC, Los \\ Angeles, CA, USA
}

Correspondence: Frank A Petrigliano USC Epstein Family Center for Sports Medicine at Keck Medicine of USC, San Pablo st\#2000, Los Angeles, CA, 90033, USA

Tel + I 310 403-044 I

Email fpetrigliano@gmail.com

\begin{abstract}
Ulnar collateral ligament (UCL) reconstruction has been successfully utilized to treat symptomatic UCL insufficiency in overhead athletes. Despite the overall success of the procedure, attempts have been made to improve upon the original technique with the goal of hastening return to sport. Most recently, there has been interest in repairing or reconstructing the native ligament with internal brace (IB) augmentation. Biomechanical cadaveric studies assessing UCL repair with IB augmentation have attempted to evaluate the efficacy of this treatment; however, the literature is seemingly divided on its benefit. Preliminary clinical studies suggest internal bracing may allow a faster return to sport than conventional techniques. The purpose of this review was to provide an analysis of the current evidence on IB augmentation in UCL repair of the elbow as it pertains to biomechanical advantages/ disadvantages, reported surgical techniques, and clinical outcomes in comparison with traditional UCL reconstruction techniques.
\end{abstract}

Keywords: return to sport, elbow, internal brace, UCL

\section{Background}

Serving as the primary medial stabilizer of the elbow, the ulnar collateral ligament (UCL) is composed of three distinct bundles: the anterior, posterior, and transverse bundles. ${ }^{1,2}$ The anterior bundle, which is most commonly injured, is composed of two bands (anterior and posterior) that serve as the primary valgus stabilizer between $30^{\circ}$ and $90^{\circ}$ of elbow flexion (anterior band) and between $90^{\circ}$ and $120^{\circ}$ of flexion (posterior band). ${ }^{1,3-5}$ Chronic overuse can result in microtrauma and ultimately UCL rupture. ${ }^{6,7}$ UCL reconstruction is commonly performed in elitelevel throwers. A study found that in 2012-2013, the prevalence rate for UCL reconstruction in professional baseball players was $10 \%$, with that number increasing to $25 \%$ for MLB pitchers and $15 \%$ for minor league pitchers. ${ }^{8}$ According to data for the 2018 baseball season, the prevalence of UCL reconstruction was $13 \%$ among professional baseball players. ${ }^{9}$ This last study showed that, within a 6-year period, there was a statistically significant increase in the prevalence of UCL reconstruction among minor league pitchers from $15 \%$ in 2012 to $19 \%$ in $2018 .{ }^{9}$

UCL injury was often thought to be a career-ending injury prior to the advent of the Jobe technique of UCL reconstruction. ${ }^{7}$ The traditional Jobe technique provided a solution to a difficult problem, but there have since been numerous modifications to UCL reconstruction techniques leading to improved clinical outcomes. ${ }^{7,10,11}$ 
Internal bracing takes a different approach to elbow UCL repair, utilizing a collagen-coated suture tape to provide stability to the repaired ligament. This approach removes some of the stress applied to the ligament once it reaches a higher level of tension. ${ }^{13}$ Other benefits include the preservation of the patient's native anatomy and proprioception, as well as limited bone loss secondary to lack of bone tunneling in this procedure. ${ }^{38}$ Dugas et al's study using human cadaveric elbows demonstrated that internal brace (IB) augmentation of the UCL repair can replicate the zero-time biomechanical properties of a modified Jobe UCL reconstruction technique. ${ }^{12}$ Biomechanical cadaveric studies have not demonstrated superiority of IB augmentation compared to the traditional techniques without IB utilization. ${ }^{12-14}$ Despite the discrepancies in the biomechanical studies to date, early clinical studies have shown concrete benefits for UCL repair with IB in athletes. ${ }^{15-18}$

The aim of this review was to evaluate the biomechanical data surrounding the utilization of IB in elbow UCL procedures [repair (no graft utilization) or reconstruction (graft utilization)]. We focused on the differences in postoperative rehabilitation and outcomes of traditional UCL reconstruction versus UCL repair with IB augmentation based on the existing literature in order to facilitate surgical decision-making.

\section{Biomechanics}

Several biomechanical studies have examined the potential advantages of internal bracing in either the reconstruction or repair of the elbow UCL (Table 1). These biomechanical studies often involved the use of cadaveric elbows placed in an axial-torsion machine to test the biokinetic properties of the ulnohumeral joint following UCL repair or reconstruction. ${ }^{12-14,20-25,27}$ Of particular interest were the effects of internal bracing on gap formation and load to failure torques. ${ }^{12-14,20-25}$ When Dugas et al first introduced the novel use of IB in the repair of the UCL, they found that at small cyclic torque values, the repair group with the use of IB was more resistant to gap formation compared to a modified Jobe reconstruction. ${ }^{12}$ At higher torque values, the repair with IB augmentation offered no statistical differences in gap formation, torsional stiffness, or torque to failure when the two techniques were compared. ${ }^{12}$ Later studies also compared the biomechanical properties of the elbow UCL reconstruction and repair with IB augmentation to traditional elbow UCL reconstruction or repair techniques. ${ }^{13,14}$ Bodendorfer et al compared the docking reconstruction technique to repair with
IB and similarly discovered that IB augmentation did not significantly affect gap formation, valgus opening, or load to failure torque. ${ }^{14}$ Bernholt et al compared UCL reconstruction with and without internal brace augmentation and found that IB augmentation did not significantly affect gap formation. ${ }^{13}$ These studies revealed that the addition of IB augmentation in either the repair or reconstruction elbow UCL could result in zero-time performance similar to traditional reconstruction techniques, providing a biomechanical argument for internal bracing as a potentially viable alternative or augmentation to traditional techniques.

In contrast, several other studies have found increased resistance to gap formation in the IB repair or reconstruction of the elbow joint, supporting the idea that internal bracing can improve zero-time biomechanics. ${ }^{21,23,24}$ Jones et al found that UCL repair with IB augmentation had significantly less gap formation than the modified Jobe UCL reconstruction at cyclic valgus rotations between $2 \mathrm{~N} \cdot \mathrm{m}$ and $10 \mathrm{~N} \cdot \mathrm{m} .{ }^{23}$ Similarly, the study by Leasure et al confirmed that a modified docking reconstruction technique augmented with internal bracing resulted in reduced gap formation when compared to a modified docking reconstruction technique. ${ }^{24}$ Bachmaier et al not only corroborated that repair with IB improved gap formation but also found that at higher valgus forces, IB augmentation led to higher torsional resistance and loading capability. ${ }^{21}$

The biomechanical literature also provided differing results regarding the advantages that internal bracing can offer to the failure torque of the repaired or reconstructed elbow UCL. ${ }^{12-14,20-25}$ While multiple studies did not observe a statistically significant difference in the load to failure torques when comparing traditional elbow UCL reconstruction techniques to either UCL repair with internal bracing ${ }^{12,14,23}$ or to UCL hybrid reconstruction with internal bracing, ${ }^{24}$ Urch et al reported that the 3-strand docking technique showed significantly increased torque to failure values compared to repair with $\mathrm{IB}^{20}$ Alternatively, some studies found that IB augmentation of the elbow UCL improved the failure strength of the elbow joint when compared to the UCL standard docking reconstruction or simple humeral repair of the UCL, respectively. ${ }^{13,22}$ Melbourne et al conducted a study that compared four groups: repair with and without IB, and reconstruction with and without IB. Their findings revealed that internal bracing in both reconstruction and repair significantly increased the load to failure torques, highlighting the biomechanical strength provided by 


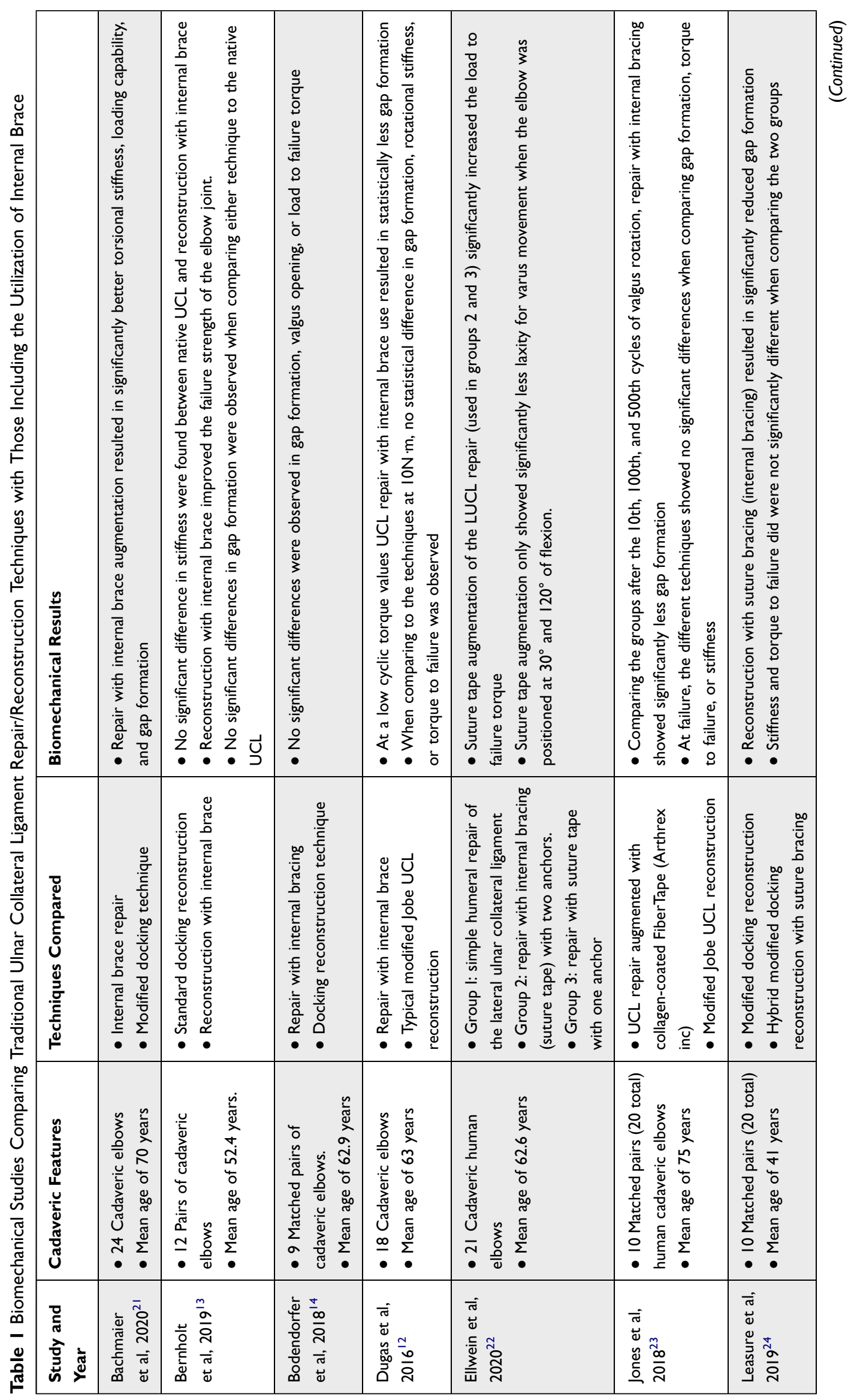




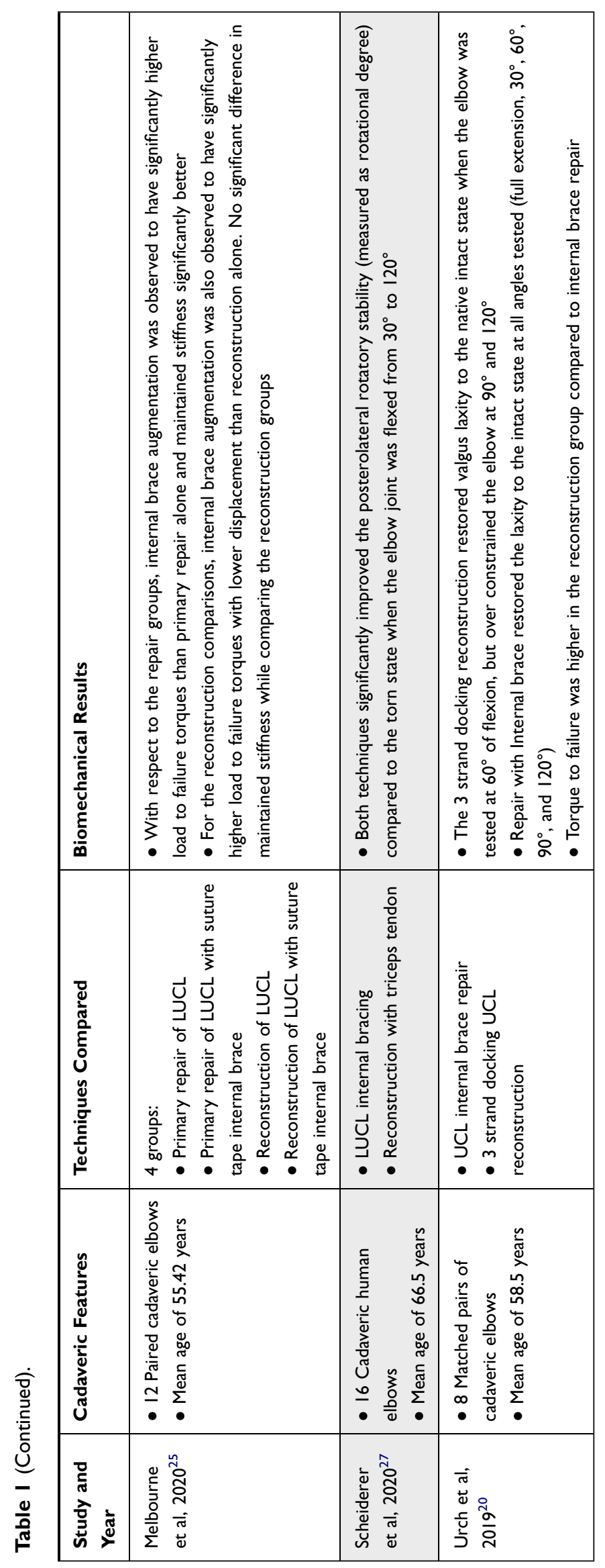


internal bracing. ${ }^{25}$ Only three studies reviewed in this paper performed a direct comparison of traditional reconstruction techniques to reconstruction with internal bracing. ${ }^{13,24,25}$ All three studies reported an advantage in the use of internal bracing in elbow UCL reconstruction, either in improving failure strength ${ }^{13,25}$ or reducing gap formation. $^{24}$ While these cadaveric studies provided a biomechanical argument for internal brace augmentation in the reconstruction of the elbow UCL, comparative clinical studies will be needed to further determine the efficacy of internal bracing in reconstruction.

The biomechanical studies mentioned above share similar limitations inherent to the nature of the cadaveric studies. First, the average age of the cadaveric elbows ranged from 41 to 75 years. ${ }^{23,24}$ The age of these elbows may not necessarily correspond to the age of younger athletes more prone to ulnar collateral ligament damage due to continuous stress. The ex vivo nature of cadaveric studies, which requires cadaveric preparation, freezing, and thawing for use, may also affect the physiological tension and rigidity of the ligaments tested. In the preparation of the elbows, any removal of secondary stabilizing muscles could also play a role in affecting the biomechanical properties of the elbow joint. There is also evidence suggesting that repetitive testing of cadaveric ligaments can result in increased laxity. ${ }^{26}$ This suggests that repetitive biomechanical studies can produce inaccurate results. Furthermore, the biomechanical studies only replicated immediate time zero post-operative kinetics. Lastly, only 3 of 10 biomechanical studies reported in this paper tested elbow biomechanics at various degrees of flexion. ${ }^{20,22,27}$ Future studies are required to assess elbow mechanics with a broader range of motion after UCL repair with IB.

\section{UCL Reconstruction vs UCL Repair with Internal Brace Augmentation}

The utilization of an internal brace is evolving as part of the elbow UCL repair procedure where the native ligament is reinforced using the internal brace and not necessarily utilizing tissue graft like the traditional reconstruction surgery. $^{28}$ While UCL reconstruction is considered the gold standard for maximizing RTP, UCL repair with modern techniques, particularly with internal bracing, has yielded RTP rates (87-96.7\%) comparable with reconstruction. $^{28}$ In 2019, Dr Dugas published a technique for UCL repair with collagen dipped FiberTape in overhead-throwing athletes. ${ }^{16}$ The authors suggested that in specific patient populations who did not want to endure the rehabilitation process of a full UCL reconstruction, UCL repair was satisfactory in facilitating return to sport. ${ }^{16}$

The evolution of UCL surgery in clinical practice seems to follow similar patterns to the advancements in techniques of biomechanical studies. In contrast to the biomechanical studies that included direct comparison between UCL reconstruction to UCL repair with IB augmentation, no clinical study has conducted a similar comparison. It is worth noting that the selection between a reconstruction versus repair procedure with internal brace augmentation often depends on the extent of the injury and the quality of the native UCL ligament of the elbow. Another factor to take into consideration upon procedure selection is the surgeon's expertise and availability of tissue graft or internal brace constructs.

When considering patient selection for UCL reconstruction vs repair, it is important to consult the patient to determine how quickly they desire to return to play, their level of competition, and their ultimate goals in athletics. Older patients who are less active may be less motivated to accelerate their rehabilitation and would be managed differently than a collegiate athlete injured 5 months before their season. Such an athlete could be a candidate for repair and be within a window to RTP by the time their season starts. ${ }^{26}$ Other considerations for patient selection are the health of the native tissue, location of the tear, and grade of the injury. In certain patients, an intraoperative evaluation of the tissue may be necessary to choose between reconstruction and repair. ${ }^{16}$

The introduction of internal brace in orthopaedic practice offers acceleration of postoperative rehabilitation compared to traditional reconstruction techniques. ${ }^{28}$ To our knowledge, the clinical outcomes of UCL reconstruction with versus without IB augmentation have not been reported, and this concept has only been tested in the cadaveric studies. $^{13,24,25}$ Similarly, prospective studies are needed to determine whether UCL repair with internal brace augmentation can yield outcomes comparable to reconstruction and to better understand potential differences in indications for these two procedures. To our knowledge, no previous study has compared the outcomes between patients with the same type of elbow UCL injury who underwent traditional reconstruction versus UCL repair with IB augmentation procedure, but the results of individual studies examining the outcomes of these two techniques are presented below. 
As the standard of care, the modified Jobe technique and docking technique continue to be two of the most widely used elbow UCL reconstruction techniques in professional athletes today and the baseline to which newer techniques will be compared against. In a review of UCL reconstruction of 566 major league pitchers between 2010 and 2014, Griffith et al found that the docking technique (n $=171,30.2 \%)$ and the modified Jobe technique $(\mathrm{n}=290$, $51.2 \%$ ) were used most frequently, making up $81.4 \%$ of the cohort. ${ }^{33}$ Reconstruction most commonly included the palmaris longus autograft (63.7\% of all reconstructions) followed by a gracilis autograft $(23.8 \%){ }^{33}$ For the modified Jobe technique, return to play (RTP) was $82.4 \%$, and return to same level (RSL) was $73.1 \% .^{33}$ The docking technique showed RTP to be $80.1 \%$ and RSL $73.7 \%{ }^{33}$ Despite the high rate of RTP, stability of the throwing arm remains in question. Within this cohort, $46.3 \%$ of the pitchers sustained subsequent elbow injuries, and 12.9\% required subsequent elbow surgeries. ${ }^{33}$ Rehabilitation was, on average, $436 \pm 146$ days for players who were able to RTP and 518.2 \pm 202.6 days for players who were able to return to the same level. ${ }^{33}$ In comparison to UCL repair, time to RTP was much longer for players who underwent UCL reconstruction surgeries. This was further supported by Cain et al follow-up of 733 patients status post reconstruction with the traditional UCL reconstruction surgery, or Tommy John Surgery (TJS). Their findings revealed an average return time for players was 11.6 months (range: $3-$ 72 months), and even longer for major league baseball (MLB) players at 16.8 months. ${ }^{32}$ Complications occurred in 148 patients $(20 \%)$. Similar to the aforementioned Griffith et al's study, the palmaris longus graft was the most commonly used graft, followed by a gracilis graft. ${ }^{32}$ Fifty-five patients underwent 62 subsequent elbow surgeries during the remainder of the study period (6 months-7 years). ${ }^{32}$ Conway et al also showed disappointing results, with only $68 \%$ of the athletes returning to the previous level of play; the number slightly increasing to $75 \%$ when considering MLB players. ${ }^{30}$ The postoperative program suggested by Conway et al recommended that pitchers return to competition at 12 months, pending adequate clinical markers. ${ }^{30}$ This recommendation aligns with the Cain et al timeline, allowing athletes to RTP at approximately 1 year from their surgery date. Among all UCL reconstruction, complication rates have been reported between $5.3 \%$ and $20 \% .^{32,35-37}$

Studies on repair with IB show promising results regarding the postoperative advantages of internal bracing, particularly with regards to the rehabilitation timeline. Having treated more than 350 athletes with IB repair of the UCL, Wilk's et al outlined a rehabilitation plan that was reported to allow athletes to return to play after about 5 months. ${ }^{26}$ Studies reporting on the clinical outcomes after repair with IB further support the idea that internal bracing can allow for a shorter rehabilitation time than conventional reconstruction. Dugas et al evaluated 111 overhead athletes who underwent a novel UCL repair technique with IB augmentation. ${ }^{16}$ Of these patients, $92 \%$ were able to RTP at the same or higher level of competition. The RTP mean was 6.7 months. ${ }^{16}$ In another cohort of 58 amateur overhead-throwing athletes, Dugas et al, found that $96 \%$ of the patients who wanted to return were able to RTP status post repair with internal bracing and did so in a shorter amount of time at 6.1 months. ${ }^{15}$ Moreover, $65 \%$ of the athletes were able to return in less than 6 months. ${ }^{15}$ O'Connell et al ${ }^{19,24}$ presented that internal bracing augmentation allowed $93 \%$ of their athletes (ages: 13-23) were able to return to their sport (at the same or higher level of competition) within 6 months. ${ }^{31}$ The return to play time presented by the these studies was much shorter than the return times after reconstruction presented by Griffith et al and Cain et al, though it must be noted that these latter studies included professional and elite-level athletes in contrast to the mostly amateur-level athletes receiving repair. ${ }^{32,33}$ Still, the clinical outcomes demonstrate that UCL repair with IB augmentation is a viable option for treatment of UCL injury and a promising area of future research.

Complications of repair with IB varied among the studies, but their relatively low rates were also promising. Dugas et al reported that $4.5 \%$ of the patients required a return to the operating room. ${ }^{16}$ Three patients experienced complications involving the ulnar nerve, one patient was taken to the operating room for exploration of the continued pain (revealing a retained suture that was then excised), and the last required two separate excisions of heterotopic bone. ${ }^{16}$ Another patient had a UCL tear more than 3 years status post the initial UCL repair with internal bracing and declined a reoperation. ${ }^{8}$ In another study, Dugas et al also reported that $5.2 \%$ of the pitchers required a return to the operating room, adding that two of these three were able to return to their previous level of play; they report only one failure over 3 years. ${ }^{3}$ Greiner et al reported that all of their 17 patients reported a complete return to their pre-injury level of activity, but 1 patient required revision by arthroscopic arthrolysis due to post-operative stiffness and mild heterotopic ossification. ${ }^{18}$ O'Connell reported 
that three patients in their studies required additional surgery and complications in $10 \%$ of the patients, most commonly ulnar nerve symptoms $(\mathrm{n}=3)$ and infection (stitch abscess, $\mathrm{n}=1$ and would infection, $\mathrm{n}=1){ }^{30}$

The work of Dugas et al, Greiner et al, and O'Connell show that the biomechanical focus in ulnar repair may now be a less invasive and more viable technique. Nonetheless, it should be noted that few if any studies have evaluated repair with IB augmentation on professional-level athletes. Additionally, the use of IB augmentation among UCL reconstruction, while often used in cadaveric studies, has not translated to clinical practice. It is entirely possible that in a field where UCL reconstructions are returning athletes to play at a high level, the impetus for change may not be as apparent. Additionally, the cost of an internal brace may be prohibitive to many patients, thus preventing further study into their utility. Our research has demonstrated the clinical benefit of the IB, allowing athletes to return to sport faster than ever before; future investigators should evaluate the efficacy of IB in UCL reconstruction to further benefit athletes looking to return to play after elbow UCL injury. Despite these limitations, studies on amateur players have shown outstanding results and show its capability in having players RTP successfully and in a short amount of time.

\section{Conclusion}

UCL repair with IB augmentation is an emerging surgical technique in the treatment of UCL injury. Biomechanical studies that have tested repair with internal bracing as an alternative to traditional techniques have produced mixed results - some providing evidence for the increasing resistance to gap formation and load to failure, while others demonstrating no statistical difference. Therefore, when considering patient selection for UCL reconstruction vs repair, it is important to consult the patient to determine how quickly they desire to return to play, their level of competition, and their ultimate goals in athletics. Clinical studies have evaluated UCL reconstruction and UCL repair with IB augmentation separately. While limited in its application to professional athletes, UCL repair with IB augmentation has produced encouraging results with early return to sports timelines, low rehabilitation time, and low reported rates of post-operative complications. Additional research is needed to examine the superiority and outcomes of UCL repair with IB augmentation in elite athletes compared to the traditional reconstruction procedure.

\section{Acknowledgment}

The authors would like to acknowledge The Cappo Family Research Fund.

\section{Disclosure}

None of the authors report any conflict of interest related to this study.

\section{References}

1. Erickson BJ, Harris JD, Chalmers PN, et al. Ulnar collateral ligament reconstruction: anatomy, indications, techniques, and outcomes. Sports Health. 2015;7(6):511-517.

2. Timmerman LA, Andrews JR. Histology and arthroscopic anatomy of the ulnar collateral ligament of the elbow. Am J Sports Med. 1994;22(5):667-673.

3. Callaway GH, Field LD, Deng XH, et al. Biomechanical evaluation of the medial collateral ligament of the elbow. J Bone Joint Surg Am. 1997;79(8):1223-1231.

4. Morrey BF, An KN. Articular and ligamentous contributions to the stability of the elbow joint. Am J Sports Med. 1983;11(5):315-319.

5. Schwab GH, Bennett JB, Woods GW, Tullos HS. Biomechanics of elbow instability: the role of the medial collateral ligament. Clin Orthop Relat Res. 1980;146:42-52.

6. Fleisig GS, Andrews JR, Dillman CJ, Escamilla RF. Kinetics of baseball pitching with implications about injury mechanisms. Am J Sports Med. 1995;23(2):233-239.

7. Jobe FW, Stark H, Lombardo SJ. Reconstruction of the ulnar collateral ligament in athletes. J Bone Joint Surg Am. 1986;68 (8):1158-1163.

8. Conte SA, Fleisig GS, Dines JS, et al. Prevalence of ulnar collateral ligament surgery in professional baseball players. Am J Sports Med. 2015;43(7):1764-1769.

9. Leland DP, Conte S, Flynn N, et al. Prevalence of medial ulnar collateral ligament surgery in 6135 current professional baseball players: a 2018 update. Orthop J Sports Med. 2019;7 (9):2325967119871442.

10. Rohrbough JT, Altchek DW, Hyman J, Williams RJ 3rd, Botts JD. Medial collateral ligament reconstruction of the elbow using the docking technique. Am J Sports Med. 2002;30(4):541-548.

11. Thompson WH, Jobe FW, Yocum LA, Pink MM. Ulnar collateral ligament reconstruction in athletes: muscle-splitting approach without transposition of the ulnar nerve. J Shoulder Elbow Surg. 2001;10 (2):152-157.

12. Dugas JR, Walters BL, Beason DP, Fleisig GS, Chronister JE. Biomechanical Comparison of ulnar collateral ligament repair with internal bracing versus modified jobe reconstruction. Am J Sports Med. 2016;44(3):735-741.

13. Bernholt DL, Lake SP, Castile RM, Papangelou C, Hauck O, Smith MV. Biomechanical comparison of docking ulnar collateral ligament reconstruction with and without an internal brace. J Shoulder Elbow Surg. 2019;28(11):2247-2252.

14. Bodendorfer BM, Looney AM, Lipkin SL, et al. Biomechanical comparison of ulnar collateral ligament reconstruction with the docking technique versus repair with internal bracing. Am J Sports Med. 2018;46(14):3495-3501.

15. Dugas JR, Looze Ca, Jones CM, et al. Ulnar collateral ligament repair with internal brace augmentation in amateur overhead throwing athletes. Orthop J Sports Med. 2018;6(7supp14):2325967118S00084.

16. Dugas JR, Looze CA, Capogna B, et al. Ulnar collateral ligament repair with collagen-dipped fibertape augmentation in overhead-throwing athletes. Am J Sports Med. 2019;47(5):1096-1102. 
17. Wilson WT, Hopper GP, Byrne PA, MacKay GM. Repair of the ulnar collateral ligament of the elbow with internal brace augmentation: a 5-year follow-up. BMJ Case Rep. 2018;11:1.

18. Greiner S, Koch M, Kerschbaum M, Bhide PP. Repair and augmentation of the lateral collateral ligament complex using internal bracing in dislocations and fracture dislocations of the elbow restores stability and allows early rehabilitation. Knee Surg Sports Traumatol Arthrosc. 2019;27(10):3269-3275.

19. Smith GR, Altchek DW, Pagnani MJ, Keeley JR. A muscle-splitting approach to the ulnar collateral ligament of the elbow neuroanatomy and operative technique. Am J Sports Med. 1996;24(5):575-580.

20. Urch E, Limpisvasti O, ElAttrache NS, et al. Biomechanical evaluation of a modified internal brace construct for the treatment of ulnar collateral ligament injuries. Orthop J Sports Med. 2019;7 (10):2325967119874135.

21. Bachmaier S, Wijdicks CA, Verma NN, Higgins LD, Greiner S. Biomechanical functional elbow restoration of acute ulnar collateral ligament tears: the role of internal bracing on gap formation and repair stabilization. Am J Sports Med. 2020;48(8):1884-1892.

22. Ellwein A, Füßler L, Ferle M, Smith T, Lill H, Pastor MF. Suture tape augmentation of the lateral ulnar collateral ligament increases load to failure in simulated posterolateral rotatory instability. Knee Surg Sports Traumatol Arthrosc. 2020.

23. Jones CM, Beason DP, Dugas JR. Ulnar collateral ligament reconstruction versus repair with internal bracing: comparison of cyclic fatigue mechanics. Orthop J Sports Med. 2018;6 (2):2325967118755991.

24. Leasure J, Reynolds K, Thorne M, Escamilla R, Akizuki K. Biomechanical comparison of ulnar collateral ligament reconstruction with a modified docking technique with and without suture augmentation. Am J Sports Med. 2019;47(4):928-932.

25. Melbourne C, Cook JL, Della Rocca GJ, Loftis C, Konicek J, Smith MJ. Biomechanical assessment of lateral ulnar collateral ligament repair and reconstruction with or without internal brace augmentation. JSES Int. 2020;4(2):224-230.

26. Pollock RG, Wang VM, Bucchieri JS, et al. Effects of repetitive subfailure strains on the mechanical behavior of the inferior glenohumeral ligament. J Shoulder Elbow Surg. 2000;9(5):427-435.

27. Scheiderer B, Imhoff FB, Kia C, et al. LUCL internal bracing restores posterolateral rotatory stability of the elbow. Knee Surg Sports Traumatol Arthrosc. 2020;28(4):1195-1201.
28. Erickson BJ, Bach BR Jr, Verma NN, Bush-Joseph CA, Romeo AA. Treatment of ulnar collateral ligament tears of the elbow: is repair a viable option? Orthop J Sports Med. 2017;5(1):2325967116682211.

29. Savoie FH 3rd, Trenhaile SW, Roberts J, Field LD, Ramsey JR. Primary repair of ulnar collateral ligament injuries of the elbow in young athletes: a case series of injuries to the proximal and distal ends of the ligament. Am J Sports Med. 2008;36(6):1066-1072.

30. Conway JE, Jobe FW, Glousman RE, Pink M. Medial instability of the elbow in throwing athletes. Treatment by repair or reconstruction of the ulnar collateral ligament. J Bone Joint Surg Am. 1992;74 (1):67-83

31. O'Connell RS, O’Brien M, Savoie FH III. Primary repair of ulnar collateral ligament injuries of the elbow. Oper Tech Sports Med. 2020;28(2):150735.

32. Cain EL Jr, Andrews JR, Dugas JR, et al. Outcome of ulnar collateral ligament reconstruction of the elbow in 1281 athletes: results in 743 athletes with minimum 2-year follow-up. Am J Sports Med. 2010;38 (12):2426-2434.

33. Griffith TB, Ahmad CS, Gorroochurn P, et al. Comparison of outcomes based on graft type and tunnel configuration for primary ulnar collateral ligament reconstruction in professional baseball pitchers. Am J Sports Med. 2019;47(5):1103-1110.

34. Cain EL, Mathis TP. Ulnar collateral ligament reconstruction: current philosophy in 2016. Am J Orthop (Belle Mead NJ). 2016;45(7): E534-e40.

35. Erickson BJ, Bach BR Jr, Cohen MS, et al. Ulnar collateral ligament reconstruction: the rush experience. Orthop J Sports Med. 2016;4 (1):2325967115626876.

36. Erickson BJ, Chalmers PN, Bush-Joseph CA, Verma NN, Romeo AA. Ulnar collateral ligament reconstruction of the elbow: a systematic review of the literature. Orthop J Sports Med. 2015;3 (12):2325967115618914.

37. Vitale MA, Ahmad CS. The outcome of elbow ulnar collateral ligament reconstruction in overhead athletes: a systematic review. $\mathrm{Am}$ J Sports Med. 2008;36(6):1193-1205.

38. Trofa DP, Lombardi JM, Noticewala MS, Ahmad CS. Ulnar collateral ligament repair with suture augmentation. Arthrosc Tech. 2017;7(1): e53-e56. doi:10.1016/j.eats.2017.08.052
Orthopedic Research and Reviews

\section{Publish your work in this journal}

Orthopedic Research and Reviews is an international, peer-reviewed, open access journal that focusing on the patho-physiology of the musculoskeletal system, trauma, surgery and other corrective interventions to restore mobility and function. Advances in new technologies, materials, techniques and pharmacological agents are particularly welcome. The manuscript management system is completely online and includes a very quick and fair peer-review system, which is all easy to use. Visit http://www.dovepress.com/testimonials.php to read real quotes from published authors. 